Article

\title{
Correlation Dynamics of Dipolar Bosons in 1D Triple Well Optical Lattice
}

\author{
Sangita Bera ${ }^{1}$, Luca Salasnich ${ }^{2}$ and Barnali Chakrabarti ${ }^{1,3, *}$ \\ 1 Department of Physics, Presidency University, 86/1 College Street, Kolkata 700073, India \\ 2 Department of Physics and Astronomy "Galileo Galilei", University of Padova Via Marzolo 8, \\ I-35131 Padova, Italy \\ 3 Abdus Salam International Centre for Theoretical Physics, I-34100 Trieste, Italy \\ * Correspondence: barnali.physics@presiuniv.ac.in
}

Received: 21 June 2019; Accepted: 8 July 2019; Published: 12 July 2019

check for updates

\begin{abstract}
The concept of spontaneous symmetry breaking and off-diagonal long-range order (ODLRO) are associated with Bose-Einstein condensation. However, as in the system of reduced dimension the effect of quantum fluctuation is dominating, the concept of ODLRO becomes more interesting, especially for the long-range interaction. In the present manuscript, we study the correlation dynamics triggered by lattice depth quench in a system of three dipolar bosons in a 1D triple-well optical lattice from the first principle using the multiconfigurational time-dependent Hartree method for bosons (MCTDHB). Our main motivation is to explore how ODLRO develops and decays with time when the system is brought out-of-equilibrium by a sudden change in the lattice depth. We compare results of dipolar bosons with contact interaction. For forward quench $\left(V_{f}>V_{i}\right)$, the system exhibits the collapse-revival dynamics in the time evolution of normalized first- and second-order Glauber's correlation function, time evolution of Shannon information entropy both for the contact as well as for the dipolar interaction which is reminiscent of the one observed in Greiner's experiment [Nature, 415 (2002)]. We define the collapse and revival time ratio as the figure of merit $(\tau)$ which can uniquely distinguish the timescale of dynamics for dipolar interaction from that of contact interaction. In the reverse quench process $\left(V_{i}>V_{f}\right)$, for dipolar interaction, the dynamics is complex and the system does not exhibit any definite time scale of evolution, whereas the system with contact interaction exhibits collapse-revival dynamics with a definite time-scale. The long-range repulsive tail in the dipolar interaction inhibits the spreading of correlation across the lattice sites.
\end{abstract}

Keywords: lattice depth quench; correlation dynamics; dipolar interaction

\section{Introduction}

The non-equilibrium dynamics of isolated quantum systems have gained enormous attention due to recent experiments with cold atoms in optical lattice. One-dimensional Bose gases have proven to be a versatile test bed for the study of quantum many-body physics out of equilibrium. The cold atoms in optical lattice offer precise control over many system parameters and the high resolution image technique allows probing their correlation dynamics [1,2]. Over the last few years, the forefront research in this direction characterizes many-body systems mainly with contact interaction [3-11]. The onset of thermalization caused by the interparticle interaction has been widely discussed in many contexts [4,12-17]. The necessary condition for thermalization is the statistical relaxation. Many-body systems relaxing to equilibrium can exhibit complex dynamics. In the context of the pioneering experiment of Greiner collapse-revival dynamics between the superfluid (SF) and Mott insulator $(M I)$ phase is observed in the interference pattern [6,7]. 
Although many theoretical works exist to characterize the dynamical process, less attention has been paid to probe the effect of long-range interaction in the time scale of dynamical evolution. Out of equilibrium dynamics of quantum systems for long-range interaction has been studied in detail for lattice bosons and spin [18]. The use of ultracold atoms with dipole-dipole interaction is the most popular tool to understand the physics of long-range $[17,19]$. Dipolar atoms in quasi-one-dimensional trap are most amenable experimentally and provide rich many-body physics not seen in three dimension [20-28]. The dipolar atoms in a triple well have already been explored using the Bose-Hubbard model and mean-field theory [29-35]. Strongly interacting dipolar bosons have recently been studied by multiconfigurational time-dependent Hartree method for bosons (MCTDHB) [36] and different quantum phases are addressed. It is also stressed that as the Bose-Hubbard model is unable to address the strongly interacting bosons in shallow lattice, it is indeed necessary to employ a general many-body approach.

In our present setup, we consider $N=3$ dipolar bosons in 1D triple-well optical lattice. We initially prepare the system in the $S F$ phase and quench it to the $M I$ phase by sudden increase in the lattice depth. The corresponding dynamics is simulated in the first principle by solving the time-dependent Schrödinger equation using MCTDHB method [37-39]. It is a general many-body method capable of addressing our system properly and its implementation in the MCTDH-X package [40-42] has been successfully employed in several earlier works [39,43-54]. We explore the transition from $S F$ to $M I$ phase by analyzing the normalized first- and second-order Glauber's correlation function and dynamics of Shannon information entropy. The observed collapse-revival dynamics is reminiscent of Greiner's experiment [6,7]. We redo the simulation with contact interaction, which also exhibits collapse-revival dynamics but in different time scale. We define figure of merit $\tau$ as the ratio of collapse time and revival time. For both kinds of interaction, $\tau$ does not change with the lattice depth, remaining consistent with the experimental result [55].

We also do the reverse quench - the system initially prepared in the MI phase is quenched to $S F$ phase by sudden decrease in lattice depth. We observe contrast between the dipolar and contact interaction in the reverse quench. For contact interaction, the system revives to $S F$ phase, however dipolar bosons exhibit very complicated dynamics. The huge correlation build-up within the individual lattice sites for dipolar interaction is not distributed over the whole lattice-the system does not turn back to $S F$ phase.

The paper is organized as follows. In Section 2, we discuss the Hamiltonian and numerical method to solve the time-dependent Schrödinger equation. In Section 3, we define the key quantities we discuss in our manuscript. In Section 4, we analyze the correlation dynamics for forward and reverse lattice depth quench for dipolar as well as for contact interaction. We draw the conclusion of our paper in Section 5 .

\section{Methodology}

The evolution of $N$ interacting bosons in 1D is governed by the time-dependent Schrödinger equation (TDSE)

$$
\hat{H} \psi=i \hbar \frac{\partial \psi}{\partial t} .
$$

The total Hamiltonian is

$$
\hat{H}=\sum_{i}^{N} \hat{h}\left(x_{i}\right)+\sum_{i<j=1}^{N} \hat{W}\left(x_{i}-x_{j}\right)
$$

where $\hat{h}\left(x_{i}\right)=-\frac{\hbar^{2}}{2 m} \frac{d^{2}}{d x_{i}^{2}}+V\left(x_{i}\right)$ is the one body Hamiltonian containing the kinetic energy and the external trapping potential. $\hat{W}\left(x_{i}-x_{j}\right)$ is the interaction potential between two particles at a position $x_{i}$ and $x_{j}$. To solve the TDSE (Equation (1)), the ansatz for the many-body wave function 
$\psi\left(x_{1}, \ldots, x_{N}, t\right)=\left\langle x_{1}, \ldots, x_{N} \mid \psi(t)\right\rangle$ is taken as a linear combination of time-dependent permanents with time-dependent coefficients

$$
|\psi(t)\rangle=\sum_{\vec{n}} C_{\vec{n}}(t)|\vec{n} ; t\rangle,
$$

where, in the second quantized representation,

$$
|\vec{n} ; t\rangle=\prod_{i=1}^{M}\left[\frac{\left(\hat{b}_{i}^{\dagger}(t)\right)^{n_{i}}}{\sqrt{n_{i} !}}\right]|v a c\rangle .
$$

The summation runs over all possible configurations $N_{\text {conf }}=\left(\begin{array}{c}N+M-1 \\ N\end{array}\right)$ and $\{|\vec{n} ; t\rangle=$ $\left.\left|n_{1}, n_{2}, \ldots, n_{M} ; t\right\rangle ; \sum_{i} n_{i}=N\right\} . \hat{b}_{i}^{\dagger}(t)$ creates a boson in the single particle state $\phi_{i}(x ; t)$. It is important to emphasize that, in the ansatz, both the expansion coefficient $\left\{C_{\vec{n}}(t) ; \sum_{i} n_{i}=N\right\}$ and the orbitals $\left\{\phi_{i}(x, t)\right\}_{i=1}^{M}$ that build up the permanents are time-dependent fully variationally optimized quantities. MCTDHB theory is established as the most efficient way to solve the time-dependent many-body problems of interacting bosons accurately and has been applied for a wide set of problems [39,43-54]. In the limit of $M \rightarrow \infty$, the set of permanents $\{|\vec{n} ; t\rangle\}$ spans the complete $N$ boson Hilbert space. As the permanents are time-dependent, a given degree of accuracy is reached with a shorter expansion as compared to the time-independent basis. To solve the TDSE for the wave function $|\psi(x, t)\rangle$, one needs to determine the evolution of the coefficients $\left\{C_{\vec{n}}(t) ; \sum_{i} n_{i}=N\right\}$ and orbitals $\left\{\phi_{i}(x, t)\right\}_{i=1}^{M}$ with time. Their equations of motion are derived requiring the stationarity of the action functional with respect to the variations of the time-dependent coefficients and the set of time-dependent orbitals. The set of nonlinear integro-differential equations are simultaneously solved by the recursive MCTDHB (R-MCTDHB) package [40-42]. It is stressed that a MCTDHB is much more accurate than exact diagonalization (ED) method. In ED, the time-independent basis is employed which are built up from the eigenvalues of a one-body problem and are not further optimized to take into the account the dynamics and correlations in the system. Conversely, MCTDHB uses time adaptive many-body basis set and its evolution follows from the time-dependent variational principle. Thus, the error resulting from the truncation of Hilbert space is minimized by the basis at any given instant of time. For the present study, for both the short- and long-range interactions, we keep $M=6$ orbitals to achieve convergence. Convergence is ascertained by systematically increasing the number of orbitals and observing no change in the calculated quantities such as energy and one-body density. Additionally, convergence is further assured when the occupation of the last orbital is negligible. In our present computation, we find that $M=6$ orbitals are adequate to capture the correct physics.

\section{Quantities of Interest}

The normalized $p$ th order correlation function is defined by

$$
g^{(p)}\left(x_{1}^{\prime}, \ldots, x_{p}^{\prime}, x_{1}, \ldots, x_{p} ; t\right)=\frac{\rho^{(p)}\left(x_{1}, \ldots, x_{p} \mid x_{1}^{\prime}, \ldots, x_{p}^{\prime} ; t\right)}{\sqrt{\prod_{i=1}^{p} \rho\left(x_{i} \mid x_{i} ; t\right) \rho\left(x_{i}^{\prime} \mid x_{i}^{\prime} ; t\right)}},
$$

which defines spatial $p$ th order coherence [56,57], where $\rho^{(p)}\left(x_{1}, \ldots, x_{p} \mid x_{1}^{\prime}, \ldots, x_{p}^{\prime} ; t\right)$ is the $p$ th order reduced density matrix. Although it is possible to define the $p$ th order correlation function in the momentum space, in the present manuscript, we report results only for spatial coherence. The normalized first-order correlation function $g^{(1)}$ defined as

$$
g^{(1)}\left(x_{1}^{\prime}, x_{1} ; t\right)=\frac{\rho^{(1)}\left(x_{1}^{\prime} \mid x_{1} ; t\right)}{\sqrt{\rho\left(x_{1}^{\prime} ; t\right) \rho\left(x_{1} ; t\right)}}
$$


which quantifies the degree of first-order coherence. $g^{(1)}<1$ means the visibility of interference fringes in the interference experiment is less than $100 \%$ which is referred as loss of coherence. $g^{(1)}=1$ corresponds to maximal fringe visibility and is referred to as full coherence. The strength of interaction between the particles affects the correlation; coherence is quickly lost for stronger interparticle interaction. The corresponding second-order correlation function $g^{(2)}$ is calculated as

$$
g^{(2)}\left(x_{1}, x_{2} ; t\right)=\frac{\rho^{(2)}\left(x_{1}, x_{2} ; t\right)}{\rho\left(x_{1} ; t\right) \rho\left(x_{2} ; t\right)}
$$

where $\rho^{(2)}$ is the diagonal of the two-body reduced density matrix and $\rho(x, t)$ is the one-body density. $g^{(2)}<1$ is referred to as anti-bunching effect and $g^{(2)}>1$ is termed as bunching effect. $g^{(2)}=1$ signifies that the measures of two particles at positions $x_{1}$ and $x_{2}$ are stochastically independent.

The Shannon information entropy of the one-body density in co-ordinate space is given by $S_{x}(t)=$ $-\int d x \rho(x, t) \ln [\rho(x, t)]$ and analogously in the conjugate space $S_{k}(t)=-\int d k \rho(k, t) \ln [\rho(k, t)][58,59]$. As the above distributions are related to the one-body density, they are insensitive to correlations that may be present in the many-body state $|\psi\rangle$. In MCTDHB theory, as $|\psi(t)\rangle=\sum_{\vec{n}} C_{\vec{n}}(t)|\vec{n} ; t\rangle$, we define alternative measure of information entropy using the time-dependent coefficients as

$$
S^{\text {info }}(t)=-\sum_{\bar{n}}\left|C_{\bar{n}}(t)\right|^{2} \ln \left|C_{\bar{n}}(t)\right|^{2} .
$$

We term this entropy as coefficient Shannon information entropy (C-SIE). It measures the effective number of basis sets that contribute to a given many-body state. As the mean-field state is a single configurational state only a single coefficient contributes and $S^{\operatorname{info}}(t)=0$ for all time $t, S^{\operatorname{info}}(t)$ cannot be produced in mean-field theory.

\section{Results for Quench Dynamics}

The external trapping potential (lattice potential) in the one-body term of the total Hamiltonian in Equation (2) can be written as

$$
V(x)=V \sin ^{2}(k x)
$$

where $V$ is the depth, $k=\frac{\pi}{d}$ is the lattice wave-vector, and $d$ is the periodicity of the lattice. The Hamiltonian $H$ is scaled in terms of the recoil energy $E_{R}=\hbar^{2} k^{2} / 2 m, \hbar=m=k=1$, thus rendering all terms dimensionless. The time is expressed in the units of $\frac{\hbar}{E_{R}}$ and distance is expressed in units of $\frac{1}{k}$. We set our grid to range from $x_{\min }=-\frac{3 \pi}{2}$ to $x_{\max }=\frac{3 \pi}{2}$ such that $d=3$ wells are considered. Our setup has $N=3$ bosons in three wells and filling factor $v=1$, which is the elementary building block that exhibits essential dipolar effect of bosons in optical lattice. For the dipolar interaction,

$$
\hat{W}\left(x_{i}-x_{j}\right)=\frac{g_{d}}{\left|x_{i}-x_{j}\right|^{3}+\alpha_{0}}
$$

where $g_{d}$ is the dipolar interaction strength and $\alpha_{0}$ is the short-range cut-off to avoid the divergence at $x_{i}=x_{j}$. For contact interaction,

$$
\hat{W}\left(x_{i}-x_{j}\right)=\lambda \delta\left(x_{i}-x_{j}\right),
$$

where $\lambda$ is the two-body coupling strength between the atoms [60]. The cut-off parameter $\alpha_{0}$ is chosen by the following normalization

$$
\int_{-\infty}^{\infty} \frac{1}{x^{3}+\alpha_{0}} d x=\int_{-\infty}^{\infty} \delta(x) d x
$$

For the simulation, we used the periodic boundary condition and the lower and upper limit of the integration were chosen as $x_{\min }=-4.7$ and $x_{\max }=+4.7$, respectively. 


\subsection{Forward Quench $\left(V_{i}=3.0, V_{f}=10.0\right)$}

We prepared the system in the initial state with the lattice depth $V=3.0$ and interaction strength $g_{d}=0.01$ which is a $S F$ phase exhibiting both inter- and intra-well coherence and tunneling is allowed. In the forward quench, we instantaneously increased the lattice depth to $V=10.0$ keeping $g_{d}$ fixed. The corresponding time evolution of the normalized first-order Glauber's correlation function $\left|g^{(1)}\left(x_{1}^{\prime}, x_{1} ; t\right)\right|^{2}$ is plotted in Figure 1 as a function of two spatial variables $x_{1}^{\prime}$ and $x_{1}$ for various time $t$. Initially, at time $t=0$, the correlation function remains close to unity for all $\left(x_{1}^{\prime}, x_{1}\right)$, the system is therefore fully coherent. With increase in time, as fragmentation is built up in the many-body state, the off-diagonal $\left(x_{1}^{\prime} \neq x_{1}\right)$ correlation is gradually lost. At time $t=21.0$, when the many-body state is completely fragmented, the correlation function is unity almost exclusively along the diagonal $\left(x_{1}^{\prime}=x_{1}\right)$. Away from the diagonal $\left(x_{1}^{\prime} \neq x_{1}\right)$, the correlation function $\left|g^{(1)}\left(x_{1}^{\prime}, x_{1} ; t\right)\right|^{2}$ is close to zero. The complete loss of off-diagonal correlation characterizes the fragmented MI state. However, in long-time dynamics, we observe that the system starts to build up coherence. At time $t=58.0$, both inter- and intra-well coherence is regained $\mathrm{ND}\left|g^{(1)}\left(x_{1}^{\prime}, x_{1} ; t\right)\right|^{2}$ becomes unity for all $\left(x_{1}^{\prime}, x_{1}\right)$-the system revives to $S F$ phase.

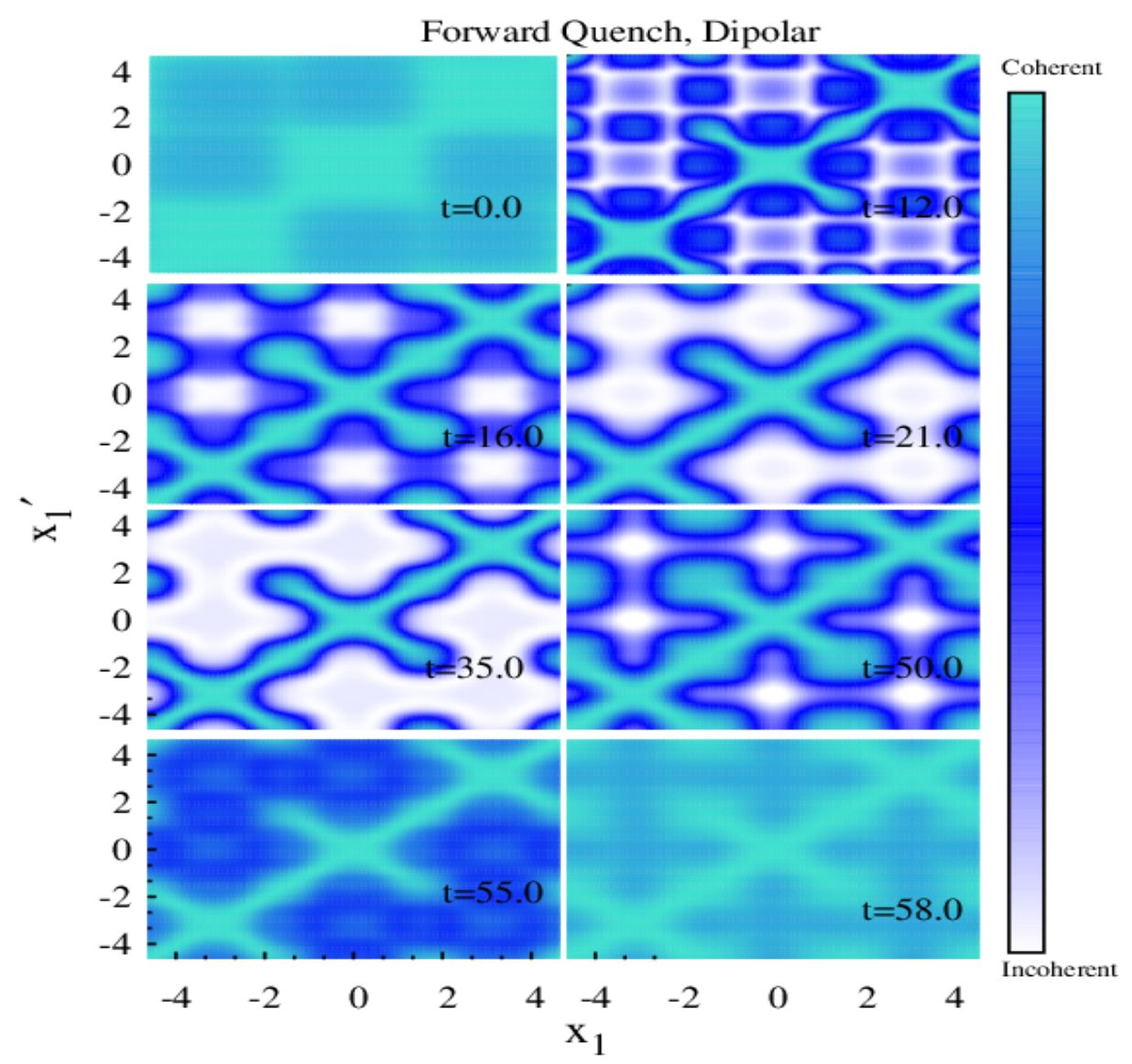

Figure 1. Time evolution of the normalized first-order Glauber's correlation function $\left|g^{(1)}\left(x_{1}^{\prime}, x_{1} ; t\right)\right|^{2}$ for forward lattice depth quench from $V_{i}=3.0$ to $V_{f}=10.0$ for dipolar interaction. We observe collapse $(S F \rightarrow M I)$-revival $(M I \rightarrow S F)$ dynamics. See the text for details. All quantities are dimensionless.

Since the coherence and correlations are experimentally accessible, it is also instructive to make a connection between the entropy dynamics and the collapse-revival cycle observed in the correlation dynamics. In Figure 2a, we plot the Shannon information entropy (calculated from Equation (8)) as a function of time. We observe a broad maxima from $t=21.0$ to $t=54.0$, which signifies that the system retains to its maximum entropy state which is a $M I$ phase. $S^{\text {info }}(t)$ goes to minimum value at $t=58.0$ - the same time when the many-body state revives to $S F$ phase as depicted in Figure 1. Thus, 
the collapse-revival cycle in coherence dynamics basically exhibits the maximum-minimum entropy cycle in the evolution of entropy. We define collapse time $t_{\text {collapse }}$ in three ways:

(a) The system enters completely fragmented MI phase.

(b) The Shannon information entropy becomes maximum.

(c) The off-diagonal correlation is completely lost.

For the present simulation, $t_{\text {collapse }}=21.0$ - uniquely determined from the above three possible ways. Similarly, revival time $t_{\text {revival }}$ can also be calculated in three ways:

(a) The system revives to SF Phase.

(b) Information entropy reaches its minimum value.

(c) The system becomes fully coherent.

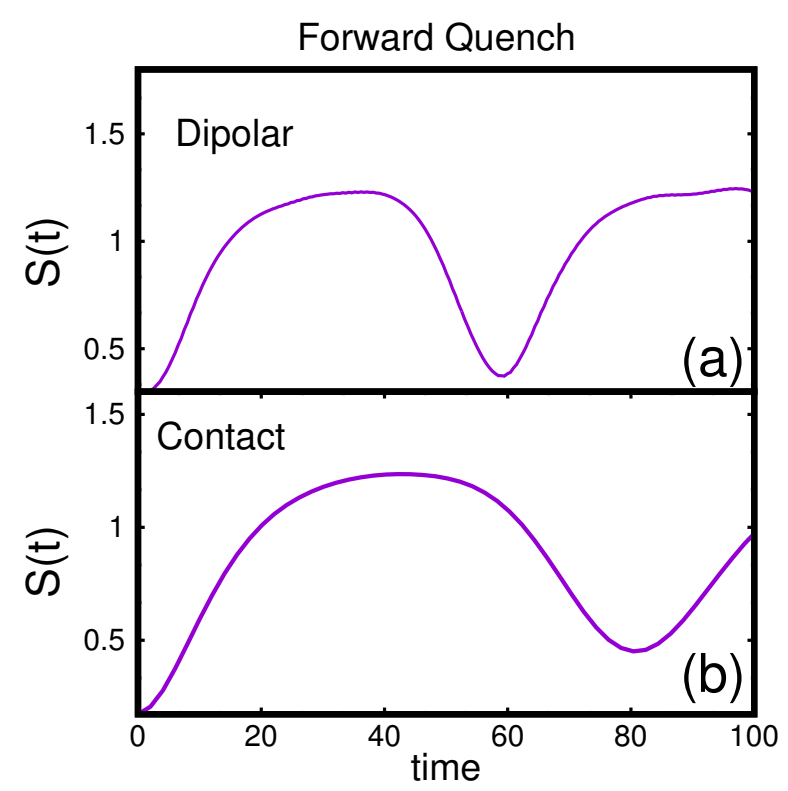

Figure 2. Time evolution of Shannon information entropy $S(t)$ for forward lattice-depth quench $V_{i}=3.0$ to $V_{f}=10.0$ : (a) dipolar interaction; and (b) contact interaction. In both cases, entropy passes through maximum point ( $M I$ phase) and minimum point (SF phase) in their corresponding time scale. All quantities are dimensionless.

For the present simulation, $t_{\text {revival }}=58.0$ was again uniquely determined by the above three criteria.

The corresponding normalized second-order Glauber's correlation function $g^{(2)}\left(x_{1}, x_{2} ; t\right)$ for dipolar interaction is shown in Figure 3 for different times. Initially, the system maintains second-order coherence for almost all $\left(x_{1}, x_{2}\right)$. Over time, the diagonal coherence starts to fade out-the probability of detecting two particles along the diagonal decreases with time. Complete deletion of the diagonal is termed as anti-bunching effect-which appears at longer time $(t=35.0)$. However, at some longer times, the system regains second-order coherence. When we repeated the quench with several larger values of lattice depth, we observed the collapse-revival dynamics is maintained. As $t_{\text {collapse }}$ and $t_{\text {revival }}$ change in individual quench process, we define the collapse to revival time ratio as a figure of merit $(\tau)$. In Figure $4 \mathrm{a}$, we plot $\tau$ as a function of lattice depth and we observe that $\tau$ is independent of the depth of the lattice potential. This observation is in agreement with previous experimental results (Figure 2 of Ref. [55]). 


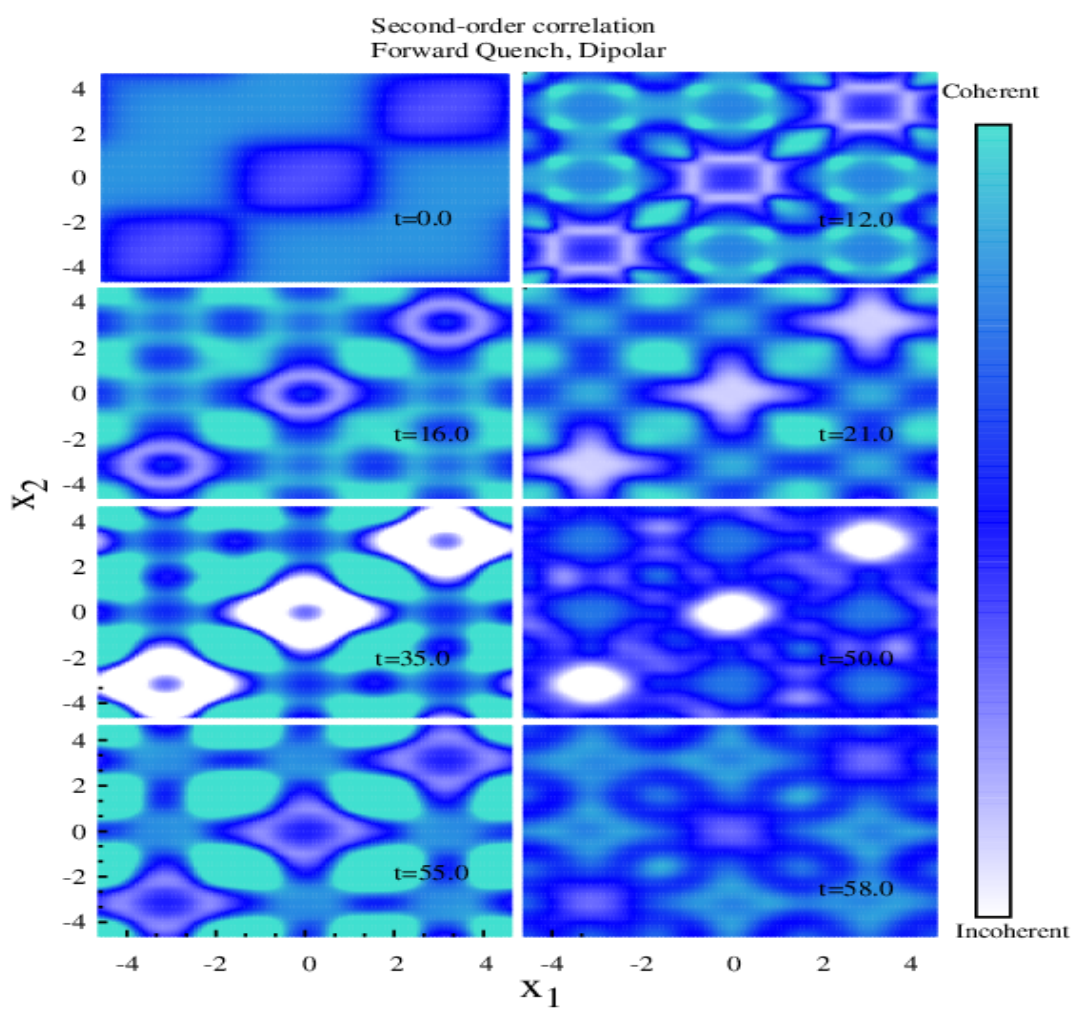

Figure 3. Time evolution of the normalized second-order Glauber's correlation function $g^{(2)}\left(x_{1}, x_{2} ; t\right)$ for forward lattice depth quench from $V_{i}=3.0$ to $V_{f}=10.0$ for dipolar interaction. We observe collapse-revival dynamics (see text). All quantities are dimensionless.

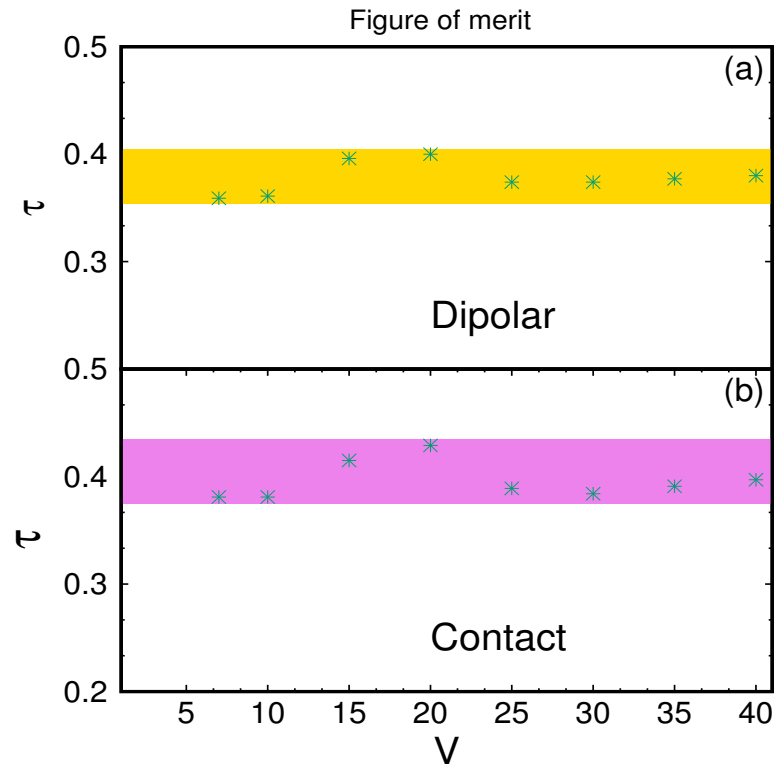

Figure 4. The ratio of collapse time $t_{\text {collapse }}$ to revival time $t_{\text {revival }}$ for different lattice depth quench:1 (a) for dipolar interaction where the yellow shading has the value $0.379 \pm 0.026$, which is bit higher than the theoretical prediction of $0.128 \pm 0.002$ for Poisson number distribution (Equation (11) of Ref. [55]); and (b) for contact interaction, where the purple shading has the width of $0.405 \pm 0.03$, which is bit higher than the prediction given above. In both cases, $\tau$ is independent of lattice depth.

To compare the observed dynamics and its time scale, we repeated the simulation for contact interaction. We observe that s similar collapse-revival dynamics is seen in the first-order coherence (Figure 5), the system passes from maximum to minimum entropy state (Figure $2 \mathrm{~b}$ ) and the 
anti-bunching effect is also developed in the second-order correlation function (Figure 6). However the time scale for contact interaction is much longer. For example, for the same simulation reported in Figures $1-3$, we observe $t_{\text {collapse }}=31.0$ and $t_{\text {revival }}=81.0$ for contact interaction in Figures 5 and 6 . Similar to dipolar interaction, we redid various lattice depth quenches for contact interaction and calculate $\tau$. We plot $\tau$ as a function of lattice depth $V$ in Figure $4 \mathrm{~b}$, which lies within the same band as depicted for dipolar interaction in Figure 4a.

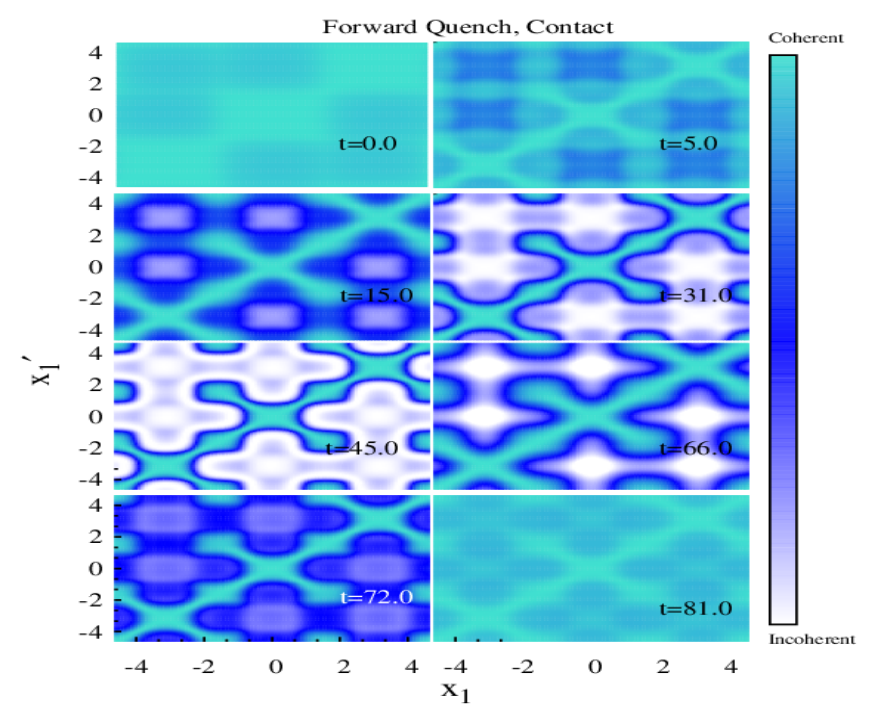

Figure 5. Time evolution of the normalized first-order Glauber's correlation function $\left|g^{(1)}\left(x_{1}^{\prime}, x_{1} ; t\right)\right|^{2}$ for forward lattice depth quench from $V_{i}=3.0$ to $V_{f}=10.0$ for contact interaction. We observe collapse-revival dynamics (see text). All quantities are dimensionless.

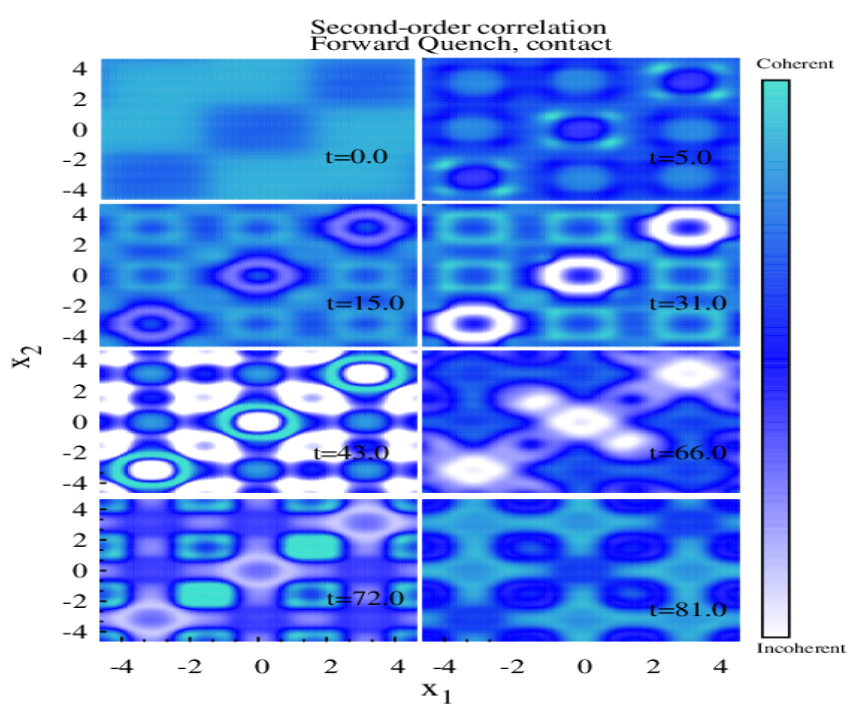

Figure 6. Time evolution of the normalized second-order Glauber's correlation function $g^{(2)}\left(x_{1}, x_{2} ; t\right)$ for forward lattice depth quench from $V_{i}=3.0$ to $V_{f}=10.0$ for contact interaction. We observe collapse-revival dynamics (see text). All quantities are dimensionless.

\subsection{Reverse Quench $\left(V_{i}=10.0\right.$ to $\left.V_{f}=3.0\right)$}

The dynamics of dipolar bosons becomes more interesting if we do the reverse quench. Now, we prepare the initial state with lattice depth $V=10.0$ and interaction strength $g_{d}=0.1$, which is a $M I$ phase-strong intra-well coherence is maintained. In this reverse quench, we fix the interaction strength and the lattice depth is instantaneously lowered to $V=3.0$. The corresponding dynamics are presented in Figures 7-9. At $t=0$, the normalized first-order Glauber's correlation function 
$\left|g^{(1)}\left(x_{1}^{\prime}, x_{1} ; t\right)\right|^{2}$ exhibits three separated lobes along the diagonal (Figure 7). Thus, $\left|g^{(1)}\left(x_{1}^{\prime}, x_{1} ; t\right)\right|^{2}$ is unity along $x_{1}^{\prime}=x_{1}$ and it is zero for all $\left(x_{1}^{\prime} \neq x_{1}\right)$. It signifies that first-order coherence is maintained within the wells and inter-well coherence is zero-it is a MI state. In the reverse quench process, the already built up intra-well coherence should be distributed across the lattice as tunneling is allowed. However, Figure 7 shows that first-order coherence exhibits complex dynamics-the system tries to build up off-diagonal correlation through inter-well tunneling but $S F$ phase is not achieved. When we performed long-time dynamics-the system passes through complex pictures and SF phase is not regained. The corresponding entropy evolution presented in Figure 8a also shows complex dynamics - the maximum or minimum entropy points are not clear. The corresponding normalized second-order Glauber's correlation function shown in Figure 9 also exhibits that strong anti-bunching effect of the initial state retains with time. Figures 7-9 uniquely exhibit the effect of long-range repulsive tail of dipolar interaction which inhibits the spreading of correlation throughout the lattice sites.

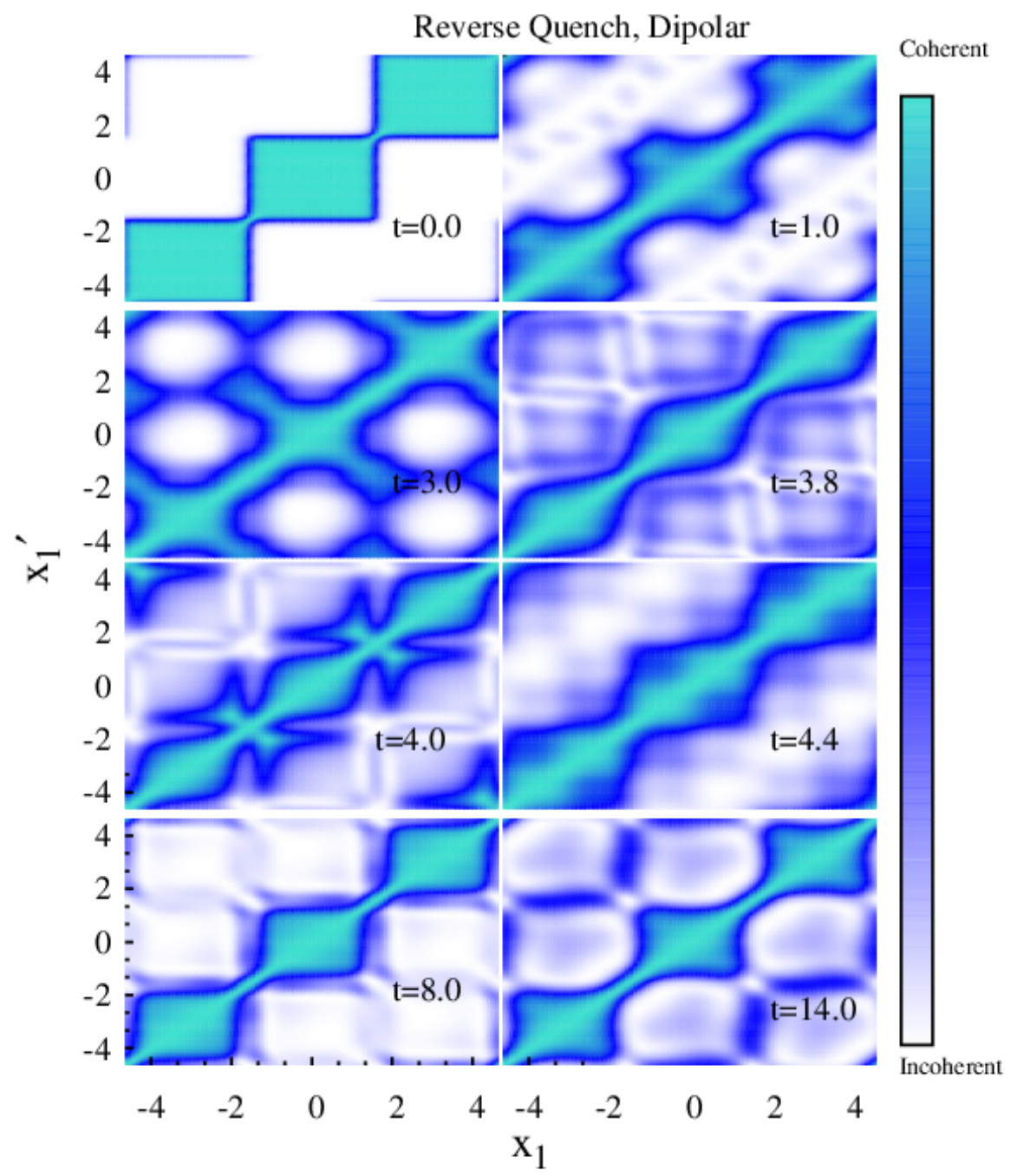

Figure 7. Time evolution of the normalized first-order Glauber's correlation function $\left|g^{(1)}\left(x_{1}^{\prime}, x_{1} ; t\right)\right|^{2}$ for reverse lattice depth quench from $V_{i}=10.0$ to $V_{f}=3.0$ for dipolar interaction. The initial Mott phase builds up some faded off-diagonal correlation, but due to long-range repulsive tail, $S F$ phase is not achieved. All quantities are dimensionless. 


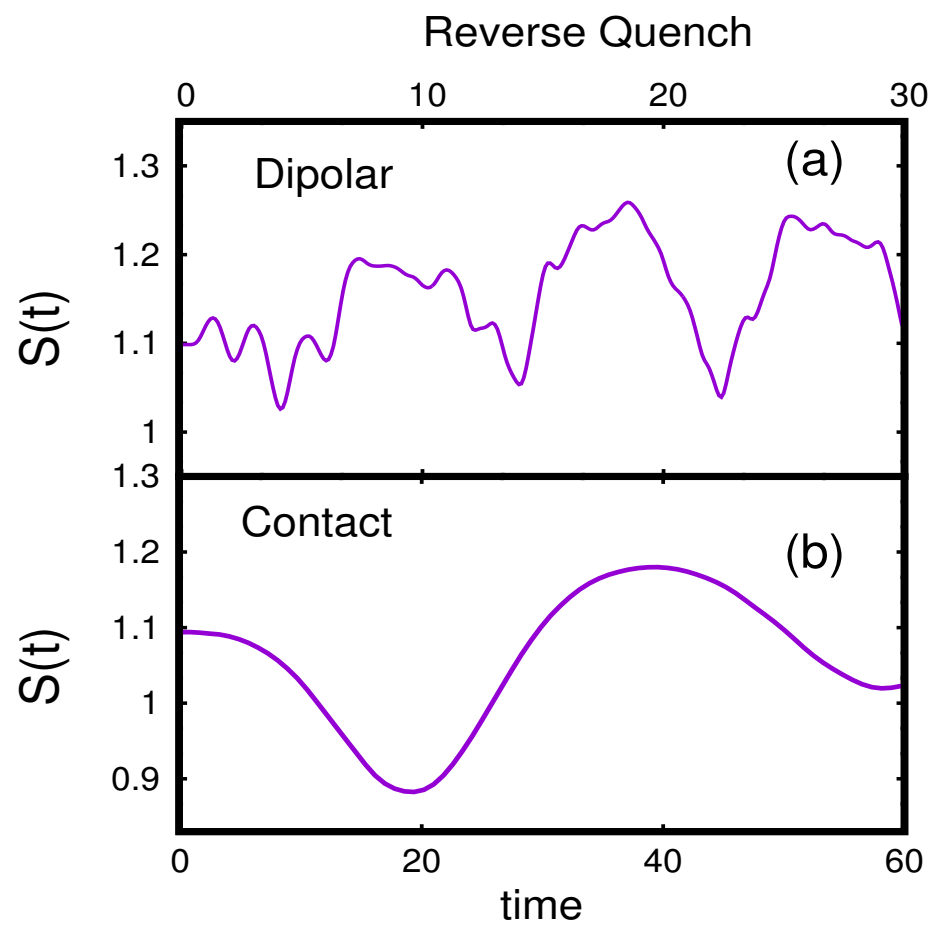

Figure 8. Time evolution of Shannon information entropy $S(t)$ for reverse lattice-depth quench $V_{i}=10.0$ to $V_{f}=3.0$ : (a) dipolar interaction and entropy shows complex dynamics; and (b) contact interaction and the entropy exhibits periodic oscillation. All the quantities are dimensionless.

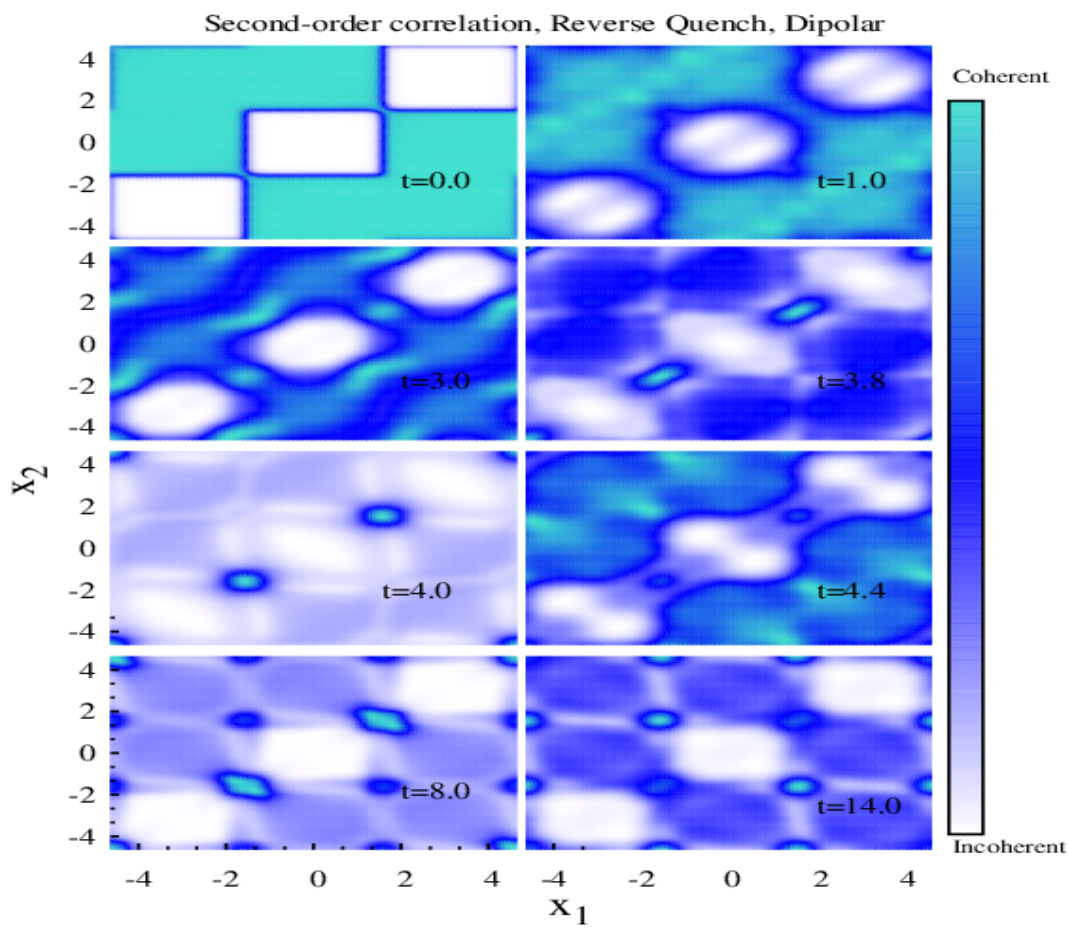

Figure 9. Time evolution of the normalized second-order Glauber's correlation function $g^{(2)}\left(x_{1}, x_{2} ; t\right)$ for reverse lattice depth quench from $V_{i}=10.0$ to $V_{f}=3.0$ for dipolar interaction (see text for details). All quantities are dimensionless.

It is also interesting to compare the reverse quench dynamics for the contact interaction. The corresponding normalized first-order Glauber's correlation function, entropy dynamics and normalized second-order Glauber's correlation function are presented in Figures $8 b, 10$ and 11, 
respectively. All of them exhibit collapse-revival dynamics. In Figure 10, we observe that initial Mott phase with exclusive diagonal correlation gradually builds up off-diagonal correlation-it is a SF phase. At some later time, MI phase comes back. In Figure 8b, we also observe that, in the revival-collapse cycle-entropy passes through maximum-minimum points. The normalized second-order Glauber's correlation function shown in Figure 11 also exhibits the revival-collapse scenario. Initially, the system has strong anti-bunching effect across the diagonal, which gradually disappears with time and the system becomes fully second-order coherent. At a later time, the anti-bunching effect develops again.

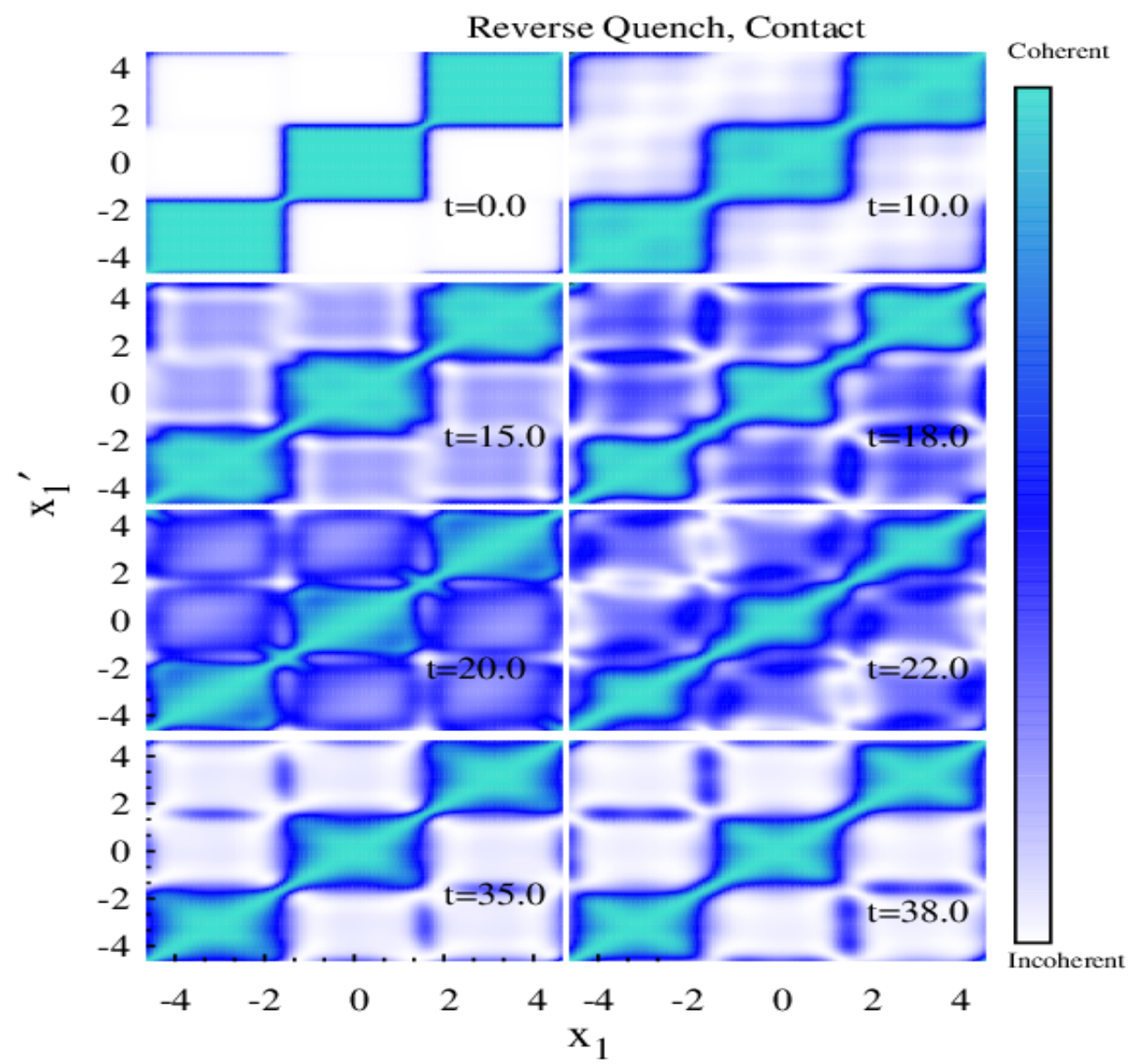

Figure 10. Time evolution of the normalized first-order Glauber's correlation function $\left|g^{(1)}\left(x_{1}^{\prime}, x_{1} ; t\right)\right|^{2}$ for reverse lattice depth quench from $V_{i}=10.0$ to $V_{f}=3.0$ for contact interaction. The initial Mott phase quickly develops off-diagonal correlation throughout the whole lattice and the correlation function for $t=20.0$ depicts $S F$ phase. At $t=38.0$ the system again enters $M I$ phase. All quantities are dimensionless.

Thus, our main observations: (1) The forward quench dynamics both for contact as well as dipolar interaction can be distinguished in their relevant time scale. The long-range repulsive tail of dipolar interaction makes the effective interaction more repulsive, which corresponds to faster time scale dynamics for dipolar bosons. (2) However, we observe contrast behavior in the reverse quench between the contact and dipolar interaction. Although for contact interaction we observe that initial Mott phase at some later time revives to $S F$ phase, for dipolar interaction, the dynamics is very complicated. We are unable to define any time scale of dynamics; the system does not come back to $S F$ phase even in the quite long-time simulation. 


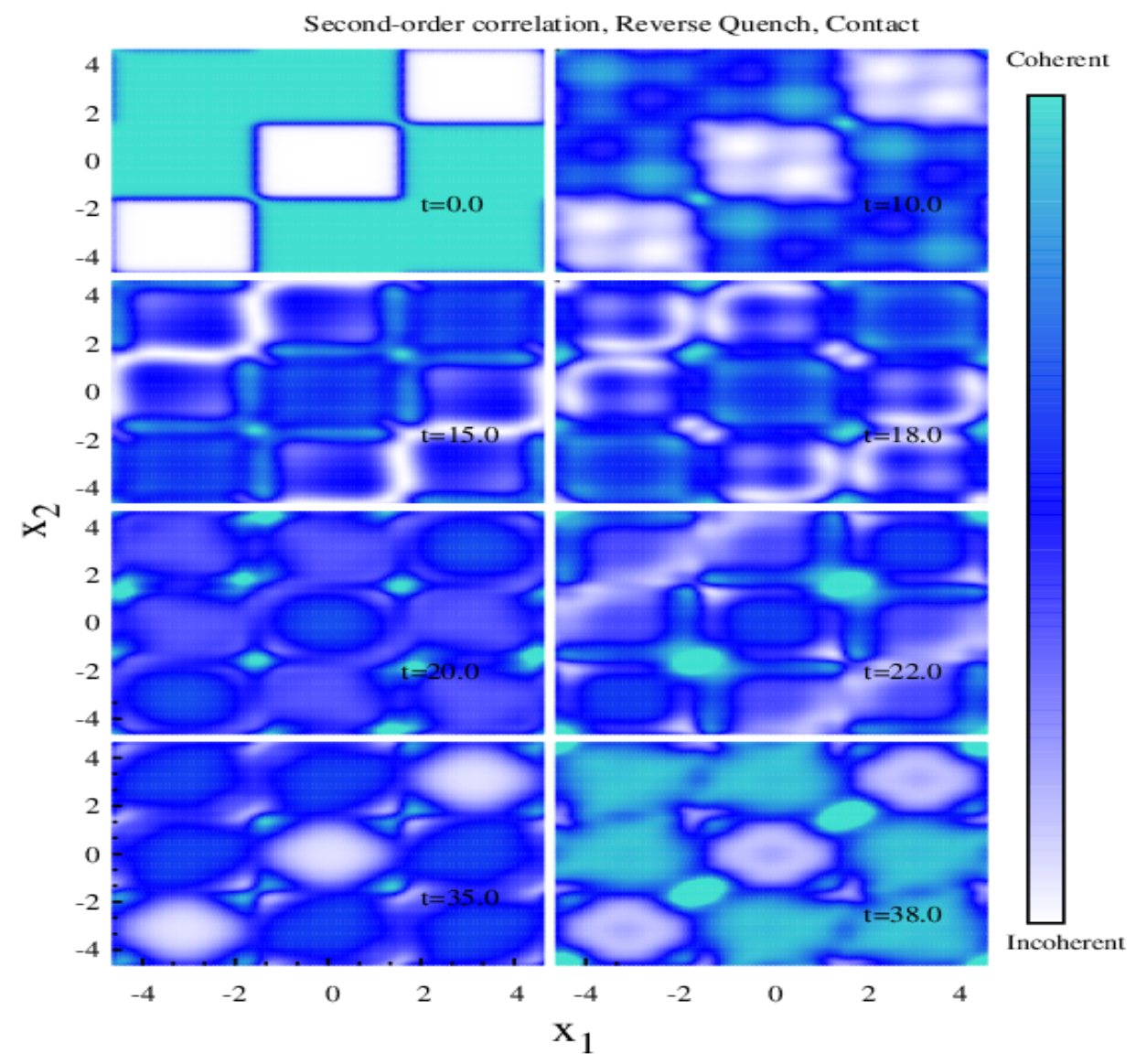

Figure 11. Time evolution of the normalized second-order Glauber's correlation function $g^{(2)}\left(x_{1}, x_{2} ; t\right)$ for reverse lattice depth quench from $V_{i}=10.0$ to $V_{f}=3.0$ for contact interaction (see text for details). All quantities are dimensionless.

The absence of revival in the reverse quench for dipolar interaction can further be explained in terms of population in natural orbitals, as shown in Figure 12b. For the forward quench, the initial SF phase is characterized by close to $100 \%$ population in the first orbital (Figure 12a). With time, the system fragments: at $t=t_{\text {collapse }}$, the system shows three-fold fragmentation, which is a Mott phase; and, at $t=t_{\text {revival }}$, the first orbital again becomes $100 \%$ populated. Thus at distinct time $S F$ to $M I$ and then $M I$ to $S F$ transition can be identified from the natural orbital occupation. In contrast, for reverse quench process, the system is initially three-fold fragmented; with time the first orbital starts to be more populated with equal decrease in the population in the other two orbitals. However, the maximum population in the first orbital, as observed in Figure 12b, is close to $50 \%$, thus $S F$ state is not achieved. Thus, the initial Mott phase remains in the Mott phase, and only the diagonal correlation spreads throughout the lattice in a complicated way. However, as not enough off-diagonal correlation is built up, $S F$ phase is not revived.

Our present simulation is based on the simplest problem of three bosons in three wells, which is the building block of many-body physics. The immediate question in this direction is to investigate the finite size effect keeping the density fixed. We repeated our simulation for $N=5$ bosons in five wells keeping filling factor $v=1$. We observed identical dynamics as reported for three bosons in three wells. If we increased the particle number to a sufficiently large value, then convergence of the measured quantity would be a serious issue. Increasing the particle number requires more and more orbitals to give converged results, which in turn will increase the size of the Hilbert space and numerical simulation will not be possible. 


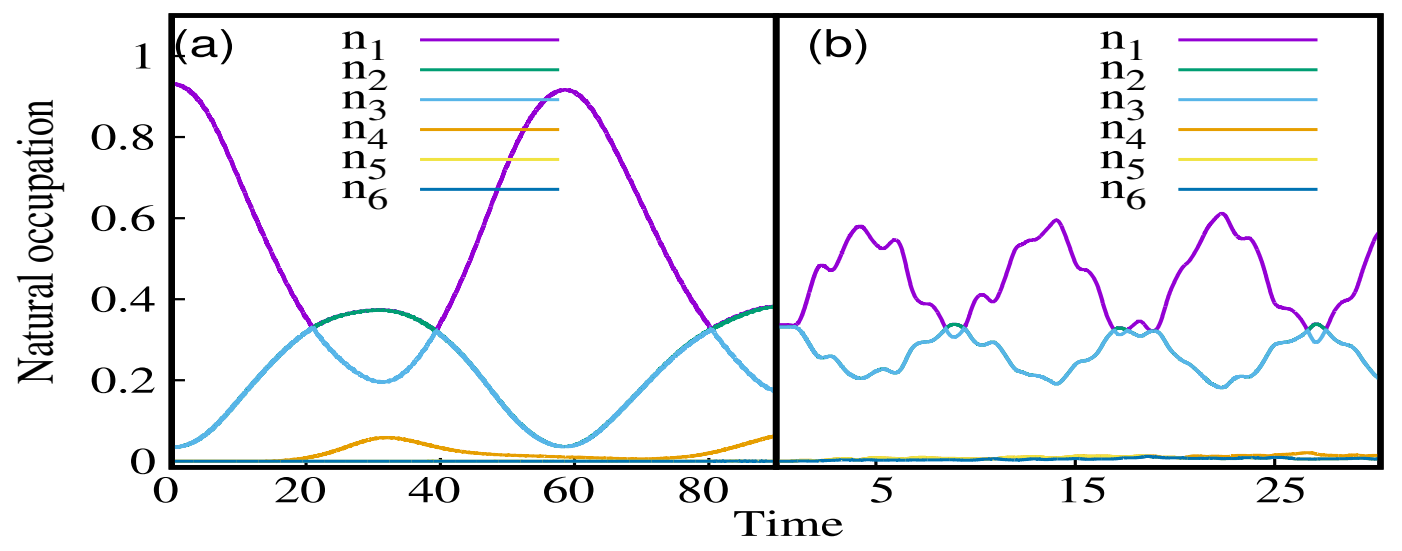

Figure 12. Time evolution of natural orbitals for dipolar interaction: (a) forward lattice-depth quench $V_{i}=3.0$ to $V_{f}=10.0$; and (b) reverse lattice-depth quench $V_{i}=10.0$ to $V_{f}=3.0$ (see text for details). All quantities are dimensionless.

\section{Conclusions}

In this paper, we study the quench dynamics of $1 \mathrm{D}$ dipolar bosons in a triple-well optical lattice from the first principle general quantum many-body perspective utilizing the MCTDHB method for both shallow and deep optical lattices. The comparison with the contact interaction is also presented. For forward lattice depth quench for both the contact and dipolar interaction, we observe collapse-revival dynamics in the time evolution of normalized first-order Glauber's correlation function. The observed dynamics is further linked with the production of many-body Shannon information entropy. However, both the collapse and revival time reported for dipolar interaction are significantly smaller than those corresponding to the contact interaction. We define figure of merit $\tau$ as the ratio of collapse time to revival time, which remains constant for different lattice depth quench. For the reverse quench, the system with contact interaction again passes through several SF or MI phases which are determined as collapse-revival cycle in the entropy dynamics. However, for dipolar interaction, the initial Mott phase has strong intra-well coherence, which is not distributed across the lattice during its time evolution. Thus, the system exhibits very complex dynamics and the initial Mott phase does not revive SF phase-it is the effect of long-range repulsive tail of the dipolar interaction.

The present manuscript mainly focuses on the contrast response of contact and dipolar interaction for reverse lattice depth quench in 1D optical lattice. Although many published works report the non-equilibrium quench dynamics for contact interaction, the same for dipolar interaction are few. Recently, Cevolani et al. [18] reported out-of-equilibrium dynamics for long-range interaction. They considered the global interaction quench where both the initial and final states are SF. They found that the long-range Bose-Hubbard model exhibits the same qualitative behavior as the short-range case. However, our present manuscript reports both the forward and reverse lattice depth quench where the initial state is $S F$ and $M I$, respectively. Although our observation for the forward quench is qualitatively the same as reported in [18], we conclude that reverse quench dynamics for long-range interaction contrast with that for contact interaction, which is not observed yet.

Author Contributions: S.B. conducted "conceptualization", "methodology", "formal analysis", "writing-original draft preparation" "writing-review and editing", "resources", "data curation" and "funding acquisition". L.S. contributed to write the "original draft preparation", "writing-review and editing","investigation", "resources", "funding acquisition". B.C. contributed to create the manuscript by giving the "original concept", "methodology", "formal analysis", "writing—original draft preparation" "writing-review and editing", "investigation", "resources" also "funding acquisition".

Funding: This research was funded by Department of Science and Technology Govt. of India, India and Name of the fund-DST INSPIRE fellowship [2015/IF150245]. Fund received from Abdus Salam International Centre for Theoretical Physics, Trieste, Italy to complete this research. 
Acknowledgments: Sangita Bera wants to acknowledge Department of Science and Technology Govt. of India, for the financial support through DST INSPIRE fellowship [2015/IF150245]. Barnali Chakrabarti acknowledges ICTP support where a major portion of the work has been done.

Conflicts of Interest: The authors declare no conflict of interest.

\section{References}

1. Bakr, W.S.; Peng, A.; Tai, M.E.; Ma, R.; Simon, J.; Gillen, J.; Foelling, S.; Pollet, L.; Greiner, M. Probing the Superfluid to Mott Insulator Transition at the Single Atom Level. Science 2010, 329, 547. [CrossRef] [PubMed]

2. Hung, C.-L.; Zhang, X.; Ha, L.-C.; Tung, S.-K.; Gemelke, N.; Chin, C. Extracting density-density correlations from in situ images of atomic quantum gases. New. J. Phys. 2011, 13, 075019. [CrossRef]

3. Trotzky, S.; Chen, Y.-A.; Flesch, A.; McCulloch, I.P.; Schollwöck, U.; Eisert, J.; Bloch, I. Probing the relaxation towards equilibrium in an isolated strongly correlated one-dimensional Bose gas. Nat. Phys. 2012, 8, 325. [CrossRef]

4. Cheneau, M.; Barmettler, P.; Poletti, D.; Endres, M.; Schaua, P.; Fukuhara, T.; Gross, C.; Bloch, I.; Kollath, C.; Kuhr, S. Light-cone-like spreading of correlations in a quantum many-body system. Nature 2012, 481, 484. [CrossRef] [PubMed]

5. Kinoshita, T.; Wenger, T.; Weiss, D.S. A quantum Newton's cradle. Nature 2006, 440, 900. [CrossRef] [PubMed]

6. Greiner, M.; Mandel, O.; Esslinger, T.; Hänsch, T.W.; Bloch, I. Quantum phase transition from a superfluid to a Mott insulator in a gas of ultracold atoms. Nature 2002, 415, 39. [CrossRef] [PubMed]

7. Greiner, M.; Mandel, O.; Hänsch, T.W.; Bloch, I. Collapse and revival of the matter wave field of a Bose Einstein condensate. Nature 2002, 419, 51. [CrossRef] [PubMed]

8. Will, S.; Best, T.; Schneider, U.; Hackermuller, L.; Luhmann, D.; Bloch, I. Time-resolved observation of coherent multi-body interactions in quantum phase revivals. Nature 2010, 465, 197. [CrossRef]

9. Langen, T.; Geiger, R.; Kuhnert, M.; Rauer, B.; Schmiedmayer, J. Local emergence of thermal correlations in an isolated quantum many-body system. Nat. Phys. 2013, 9, 640. [CrossRef]

10. Nagao, K.; Kunimi, M.; Takasu, Y.; Takahashi, Y.; Danshita, I. Semiclassical quench dynamics of Bose gases in optical lattices. Phys. Rev. A 2019, 99, 023622. [CrossRef]

11. Lacki, M.; Heyl, M. Dynamical quantum phase transitions in collapse and revival oscillations of a quenched superfluid. Phys. Rev. B 2019, 99, 121107. [CrossRef]

12. Miyake, H.; Siviloglou, G.A.; Puentes, G.; Pritchard, D.E.; Ketterle, W.; Weld, D.M. Bragg Scattering as a Probe of Atomic Wave Functions and Quantum Phase Transitions in Optical Lattices. Phys. Rev. Lett. 2011, 107, 175302. [CrossRef] [PubMed]

13. Natu, S.S.; McKay, D.C.; DeMarco, B.; Mueller, E.J. Evolution of condensate fraction during rapid lattice ramps. Phys. Rev. A 2012, 85, 061601.

14. Hung, C.-L.; Gurarie, V.; Chin, C. From Cosmology to Cold Atoms: Observation of Sakharov Oscillations in a Quenched Atomic Superfluid. Science 2013, 341, 1213. [CrossRef] [PubMed]

15. Gring, M.; Kuhnert, M.; Langen, T.; Kitagawa, T.; Rauer, B.; Schreitl, M.; Mazets, I.; Smith, D.A.; Demler, E.; Schmiedmayer, J. Relaxation and Prethermalization in an Isolated Quantum System. Science 2012, 337, 1318. [CrossRef] [PubMed]

16. Fischer, U.R.; Schutzhold, R.; Uhlmann, M. Bogoliubov theory of quantum correlations in the time-dependent Bose-Hubbard model. Phys. Rev. A 2008, 77, 043615. [CrossRef]

17. Lahaye, T.; Menotti, C.; Santos, L.; Lewenstein, M.; Pfau, T. The physics of dipolar bosonic quantum gases. Rep. Prog. Phys. 2009, 72, 126401. [CrossRef]

18. Cevolani, L.; Carleo, G.; Sanchez-Palencia, L. Protected quasilocality in quantum systems with long-range interactions. Phys. Rev. A 2015, 92, 041603. [CrossRef]

19. Baranov, M.A. Theoretical progress in many-body physics with ultracold dipolar gases. Phys. Rep. 2008, 464, 71. [CrossRef]

20. Cazalilla, M.; Citro, R.; Giamarchi, T.; Orignac, E.; Rigol, M. One dimensional bosons: From condensed matter systems to ultracold gases. Rev. Mod. Phys. 2011, 83 1405. [CrossRef]

21. Bloch, I.; Dalibard, J.; Zwerger, W. Many-body physics with ultracold gases. Rev. Mod. Phys. 2008, 80, 885. [CrossRef] 
22. Langen, T. Non-Equilibrium Dynamics of One-Dimensional Bose Gases. Ph.D. Thesis, Vienna University of Technology, Vienna, Austria, 2013.

23. Zöllner, S.; Bruun, G.M.; Pethick, C.J.; Reimann, S.M. Bosonic and Fermionic Dipoles on a Ring. Phys. Rev. Lett. 2011, 107, 035301. [CrossRef] [PubMed]

24. Astrakharchik, G.E.; Morigi, G.E.; de Chiara, G.; Boronat, J. Ground state of low-dimensional dipolar gases: Linear and zigzag chains. Phys. Rev. A 2008, 78, 063622. [CrossRef]

25. Astrakharchik, G.E.; Lozovik, Y.E. Super-Tonks-Girardeau regime in trapped one-dimensional dipolar gases. Phys. Rev. A 2008, 77, 013404. [CrossRef]

26. Deuretzbacher, F.; Cremon, J.C.; Reimann, S.M. Ground-state properties of few dipolar bosons in a quasi-one-dimensional harmonic trap. Phys. Rev. A 2010, 81, 063616. [CrossRef]

27. Arkhipov, A.S.; Astrakharchik, G.E.; Belikov, A.V.; Lozovik, Y.E. Ground-state properties of a one-dimensional system of dipoles. JETP Lett. 2005, 82, 39. [CrossRef]

28. Imambekov, A.; Mazets, I.E.; Petrov, D.S.; Gritsev, V.; Manz, S.; Hofferberth, S.; Schumm, T.; Demler, E.; Schmiedmayer, J. Density ripples in expanding low-dimensional gases as a probe of correlations. Phys. Rev. A 2009, 80, 033604. [CrossRef]

29. Peter, D.; Pawlowski, K.; Pfau, T.; Rzążewski, K. Mean-field description of dipolar bosons in triple-well potentials. J. Phys. B 2012, 45, 225302. [CrossRef]

30. Lahaye, T.; Pfau, T.; Santos, L. Mesoscopic Ensembles of Polar Bosons in Triple-Well Potentials. Phys. Rev. Lett. 2010, 104, 170404. [CrossRef] [PubMed]

31. Dell'Anna, L.; Mazzarella, G.; Penna, V.; Salasnich, L. Entanglement entropy and macroscopic quantum states with dipolar bosons in a triple-well potential. Phys. Rev. A 2013, 87, 053620. [CrossRef]

32. Xiong, B.; Fischer, U.R. Interaction-induced coherence among polar bosons stored in triple-well potentials. Phys. Rev. A 2013, 88, 063608. [CrossRef]

33. Gallemi, A.; Guilleumas, M.; Mayol, R.; Sanpera, A. Role of anisotropy in dipolar bosons in triple-well potentials. Phys. Rev. A 2013, 88, 063645. [CrossRef]

34. Gallemi, A.; Queralto, G.; Guilleumas, M.; Mayol, R.; Sanpera, A. Quantum spin models with mesoscopic Bose-Einstein condensates. Phys. Rev. A 2016, 94, 063626. [CrossRef]

35. Biedroń, K.; Lacki, M.; Zakrzewski, J. Extended Bose-Hubbard model with dipolar and contact interactions. Phys. Rev. B 2018, 97, 245102. [CrossRef]

36. Chatterjee, B.; Lode, A.U.J. Order parameter and detection for a finite ensemble of crystallized one-dimensional dipolar bosons in optical lattices. Phys. Rev. A 2018, 98, 053624. [CrossRef]

37. Alon, O.E.; Streltsov, A.I.; Cederbaum, L.S. Multiconfigurational time-dependent Hartree method for bosons: Many-body dynamics of bosonic systems. Phys. Rev. A 2008, 77, 033613. [CrossRef]

38. Alon, O.E.; Streltsov, A.I.; Cederbaum, L.S. Unified view on multiconfigurational time propagation for systems consisting of identical particles. J. Chem. Phys. 2007, 127, 154103. [CrossRef] [PubMed]

39. Streltsov, A.I.; Alon, O.E.; Cederbaum, L.S. Role of Excited States in the Splitting of a Trapped Interacting Bose-Einstein Condensate by a Time-Dependent Barrier. Phys. Rev. Lett. 2007, 99, 030402. [CrossRef]

40. Fasshauer, E.; Lode, A.U.J. Multiconfigurational time-dependent Hartree method for fermions: Implementation, exactness, and few-fermion tunneling to open space. Phys. Rev. A 2016, 93, 033635. [CrossRef]

41. Lode, A.U.J. Multiconfigurational time-dependent Hartree method for bosons with internal degrees of freedom: Theory and composite fragmentation of multicomponent Bose-Einstein condensates. Phys. Rev. A 2016, 93, 063601. [CrossRef]

42. Lode, A.U.J.; Tsatsos, M.C.; Fasshauer, E.; Lin, R.; Papariello, L.; Molignini, P.; Lévêque, C. MCTDH-X: The Time-Dependent Multiconfigurational Hartree for Indistinguishable Particles Software. 2018. Available online: http:/ / ultracold.org (accessed on 1 June 2019).

43. Neuhaus-Steinmetz, J.; Mistakidis, S.I.; Schmelcher, P. Quantum dynamical response of ultracold few-boson ensembles in finite optical lattices to multiple interaction quenches. Phys. Rev. A 2017, 95, 053610. [CrossRef]

44. Koutentakis, G.M.; Mistakidis, S.I.; Schmelcher, P. Quench-induced resonant tunneling mechanisms of bosons in an optical lattice with harmonic confinement. Phys. Rev. A 2017, 95, 013617. [CrossRef]

45. Mistakidis, S.I.; Schmelcher, P. Mode coupling of interaction quenched ultracold few-boson ensembles in periodically driven lattices. Phys. Rev. A 2017, 95, 013625. [CrossRef]

46. Mistakidis, S.I.; Cao, L.; Schmelcher, P. Interaction quench induced multimode dynamics of finite atomic ensembles. J. Phys. B At. Mol. Opt. Phys. 2014, 47, 225303. [CrossRef] 
47. Mistakidis, S.I.; Wulf, T.; Negretti, A.; Schmelcher, P. Resonant quantum dynamics of few ultracold bosons in periodically driven finite lattices. J. Phys. B At. Mol. Opt. Phys. 2015, 48, 244004. [CrossRef]

48. Alon, O.E.; Streltsov, A.I.; Cederbaum, L.S. Multiorbital mean-field approach for bosons, spinor bosons, and Bose-Bose and Bose-Fermi mixtures in real-space optical lattices. Phys. Rev. A 2007, 76, 013611. [CrossRef]

49. Plaßmann, T.; Mistakidis, S.I.; Schmelcher, P. Quench dynamics of finite bosonic ensembles in optical lattices with spatially modulated interactions. J. Phys. B At. Mol. Opt. Phys. 2018, 51, 225001. [CrossRef]

50. Mistakidis, S.I.; Cao, L.; Schmelcher, P. Negative-quench-induced excitation dynamics for ultracold bosons in one-dimensional lattices. Phys. Rev. A 2015, 91, 033611. [CrossRef]

51. Nguyen, H.V.; Tsatsos, M.C.; Luo, D.; Lode, A.U.J.; Telles, G.D.; Bagnato, V.S.; Hulet, R.G. Parametric Excitation of a Bose-Einstein Condensate: From Faraday Waves to Granulation. Phys. Rev. X 2019, 9, 011052. [CrossRef]

52. Lode, A.U.J.; Chakrabarti, B.; Kota, V.K.B. Many-body entropies, correlations, and emergence of statistical relaxation in interaction quench dynamics of ultracold bosons. Phys. Rev. A 2015, 92, 033622. [CrossRef]

53. Roy, R.; Gammal, A.; Tsatsos, M.C.; Chatterjee, B.; Chakrabarti, B.; Lode, A.U.J. Phases, many-body entropy measures, and coherence of interacting bosons in optical lattices. Phys. Rev. A 2018, 97, 043625. [CrossRef]

54. Bera, S.; Chakrabarti, B.; Gammal, A.; Tsatsos, M.C.; Lekala, M.L.; Chatterjee, B. Lévêque, C.; Lode, A.U.J. Sorting Fermionization from Crystallization in Many-Boson Wavefunctions. arXiv 2018, arXiv:1806.02539.

55. Zhou, T.; Yang, K.; Zhu, Z.; Yu, X.; Yang, S.; Xiong, W.; Zhou, X.; Chen, X. Observation of atom-number fluctuations in optical lattices via quantum collapse and revival dynamics. Phys. Rev. A 2019, 99, 013602. [CrossRef]

56. Sakmann, K.; Streltsov, A.I.; Alon, O.E.; Cederbaum, L.S. Reduced density matrices and coherence of trapped interacting bosons. Phys. Rev. A 2008, 78, 023615. [CrossRef]

57. Glauber, R.J. The quantum theory of optical coherence. Phys. Rev. 1963, 130, 2529. [CrossRef]

58. Massen, S.E.; Moustakidis, C.C.; Panos, C.P. Comparison of the information entropy in fermionic and bosonic systems. Phys. Lett. A 2002, 299, 131. [CrossRef]

59. Haldar, S.K.; Chakrabarti, B.; Das, T.K.; Biswas, A. Correlated many-body calculation to study characteristics of Shannon information entropy for ultracold trapped interacting bosons. Phys. Rev. A 2013, 88, 033602. [CrossRef]

60. Olshanii, M. Atomic Scattering in the Presence of an External Confinement and a Gas of Impenetrable Bosons, Phys. Rev. Lett. 1998, 81, 938. [CrossRef]

(C) 2019 by the authors. Licensee MDPI, Basel, Switzerland. This article is an open access article distributed under the terms and conditions of the Creative Commons Attribution (CC BY) license (http:// creativecommons.org/licenses/by/4.0/). 